\title{
Análise de terminações nervosas no complexo labrum- bíceps superior usando imunohistoquímica de fluorescência e microscopia a laser confocal*
}

\section{Analysis of Nerve Endings in the Superior Labrum-Biceps Complex by using Fluorescence Immunohistochemistry and Confocal Laser Microscopy}

\author{
Maria L. C. Cavalcante ${ }^{10}$ Eduardo G. Fernandes ${ }^{10}$ José A. Pinheiro Júnior ${ }^{10}$ \\ Francisco Vagnaldo F. Jamacaru ${ }^{20}$ José Victor de V. Coelho ${ }^{10}$ José Alberto Dias Leite ${ }^{10}$ \\ 1 Departamento de Ortopedia, Universidade Federal do Ceará, \\ Hospital Universitário Walter Cantídio, Fortaleza, CE, Brasil \\ 2 Departamento de Farmacologia, Universidade Federal do Ceará, \\ Fortaleza, CE, Brasil

\begin{abstract}
Endereço para correspondência Maria Cavalcante, MD PhD, Departamento de Ortopedia, Universidade Federal do Ceará, Hospital Universitário Walter Cantídio, Rua Cap. Francisco Pedro, 1395, Fortaleza, CE, 60430-140, Brasil

(e-mail: marialuzetecosta@gmail.com).
\end{abstract}

Rev Bras Ortop 2022;57(5):863-867.

\section{Resumo}

\section{Palavras-chave}

- bíceps

- técnica de anticorpos fluorescentes

- labrum

- mecanorreceptores

- terminações nervosas

- ombro
Objetivos As estruturas capsulo-ligamentares do ombro funcionam como estabilizadores estáticos, juntamente com os músculos do bíceps e do manguito rotador, aumentando a superfície de contato da cavidade glenoide. Terminações nervosas livres e mecanorreceptores foram identificados no ombro; no entanto, existem alguns estudos que descrevem a presença desses nervos na inserção do bíceps. Este estudo teve como objetivo descrever a morfologia e distribuição de terminações nervosas utilizando imunofluorescência com protein gene product 9.5 (PGP 9.5) e microscopia confocal.

Métodos Foram estudados seis complexos labrum-bíceps de seis cadáveres congelados frescos. Os espécimes foram cortados coronalmente e preparados pelo método de imunofluorescência. Tanto em hematoxilina e eosina (H\&E) quanto em imunofluorescência, foi descrita a organização do tecido conjuntivo com fibras paralelas de colágeno.

Resultados No estudo de H\&E, foram visualizadas estruturas vasculares e algumas estruturas nervosas, que foram identificadas pela presença alongada da célula nervosa. Todas as amostras analisadas com imunofluorescência e microscopia confocal
Trabalho desenvolvido no Departamento de Ortopedia, Universidade Federal do Ceará, Hospital Universitário Walter Cantídio, Fortaleza, Ceará, Brasil. recebido

01 de Junho de 2020

aceito

16 de Setembro de 2020

Publicado on-line

Agosto 13, 2021
DOI https://doi.org/

$10.1055 / \mathrm{s}-0040-1722579$

ISSN 0102-3616. (c) 2021. Sociedade Brasileira de Ortopedia e Traumatologia. All rights reserved.

This is an open access article published by Thieme under the terms of the Creative Commons Attribution-NonDerivative-NonCommercial-License, permitting copying and reproduction so long as the original work is given appropriate credit. Contents may not be used for commercial purposes, or adapted, remixed, transformed or built upon. (https://creativecommons.org/ licenses/by-nc-nd/4.0/)

Thieme Revinter Publicações Ltda., Rua do Matoso 170, Rio de Janeiro, RJ, CEP 20270-135, Brazil 


\section{Abstract}

\section{Keywords}

- biceps

- fluorescent antibody technique

- labrum

- mechanoreceptors

- nerve endings

- shoulder demonstraram baixa ocorrência de morfotipos de corpúsculos sensoriais e terminações nervosas livres. Identificamos terminações nervosas livres localizadas no labrum, inserção bicipital e terminações nervosas esparsas ao longo do tendão. Terminais corpusculares com aspecto fusiforme, cuneiforme e oval foram identificados no tendão.

Conclusão Esses achados corroboram a hipótese de que a geração de dor nas lesões labrais superiores de anterior a posterior (SLAP, na sigla em inglês) deriva da parte mais proximal do cabo longo do bíceps e ainda mais do labrum superior. Estudos quantitativos futuros com um número maior de espécimes podem fornecer mais informações sobre esses sistemas sensoriais.

Objectives The capsuloligamentous structures of the shoulder work as static stabilizers, together with the biceps and rotator cuff muscles, increasing the contact surface of the glenoid cavity. Free nerve endings and mechanoreceptors have been identified in the shoulder; however, there are a few studies that describe the presence of these nerves in the biceps' insertion. The present study aimed to describe the morphology and distribution of nerve endings using immunofluorescence with protein gene product 9.5 (PGP 9.5) and confocal microscopy.

Methods Six labrum-biceps complexes from six fresh-frozen cadavers were studied. The specimens were coronally cut and prepared using the immunofluorescence technique. In both hematoxylin and eosin (H\&E) and immunofluorescence, the organization of the connective tissue with parallel collagen fibers was described.

Results In the H\&E study, vascular structures and some nerve structures were visualized, which were identified by the elongated presence of the nerve cell. All specimens analyzed with immunofluorescence and confocal microscopy demonstrated poor occurrence of morphotypes of sensory corpuscles and free nerve endings. We identified free nerve endings located in the labrum and in the bicipital insertion, and sparse nerve endings along the tendon. Corpuscular endings with fusiform, cuneiform, and oval aspect were identified in the tendon.

Conclusion These findings support the hypothesis that the generation of pain in the superior labral tear from Anterior to posterior (SLAP) lesions derives from the more proximal part of the long biceps cord and even more from the upper labrum. Future quantitative studies with a larger number of specimens may provide more information on these sensory systems.

\section{Introdução}

O labrum superior da glenoide serve como local de fixação da inserção do tendão da cabeça longa dos bíceps, sendo assim suscetível à lesão produzida pelo seu descolamento da glenoide, de anterior a posterior, conhecida como lesão labral superior de anterior a posterior (SLAP, na sigla em inglês). ${ }^{1}$ Andrews et al. ${ }^{2}$ foi o primeiro a descrever lesões no labrum superior. Estas podem ser observadas com frequência em jovens atletas de "esportes de arremesso", sendo secundárias a microlesões repetitivas. ${ }^{3}$ Também pode ocorrer em associação com outras situações traumáticas, como deslocamento glenoumeral primário ou recorrente. ${ }^{4}$ Clinicamente, observou-se que, após reparos anatômicos da SLAP, os pacientes apresentaram um período prolongado de dor, quando comparados aos submetidos aos procedimentos de liberação de bíceps. ${ }^{5-7} 0$ mecanismo fisiopatológico da dor, presente tanto na lesão quanto no reparo cirúrgico e na eventual falha do tratamento, tem sido envolvido na presença de nociceptores. Estudos histológicos do complexo labral demonstraram a presença de terminações nervosas livres e mecanorreceptores. ${ }^{8,9}$ Sintomas como dor, instabilidade e sensação de "travamento articular podem ser atribuídos ao enfraquecimento do reflexo proprioceptivo. ${ }^{10}$ Embora os relatos de lesões por SLAP sejam frequentes na literatura, estudos recentes têm relatado um aumento considerável no número de reparos cirúrgicos dessas lesões na última década. ${ }^{11}$ Atualmente, os avanços em marcadores de anticorpos específicos para terminações nervosas, associados à microscopia a laser confocal, permitem a visualização e detalhamento de estruturas nervosas com imagens tridimensionais. $^{12-14} \mathrm{O}$ objetivo desta investigação foi avaliar histologicamente a porção média do complexo bíceps-labral superior. Nós imaginamos que a zona de transição entre o labrum e a cabeça longa do tendão do bíceps conteria terminações nervosas e vasos. Esperamos estabelecer um paralelo entre nossos 

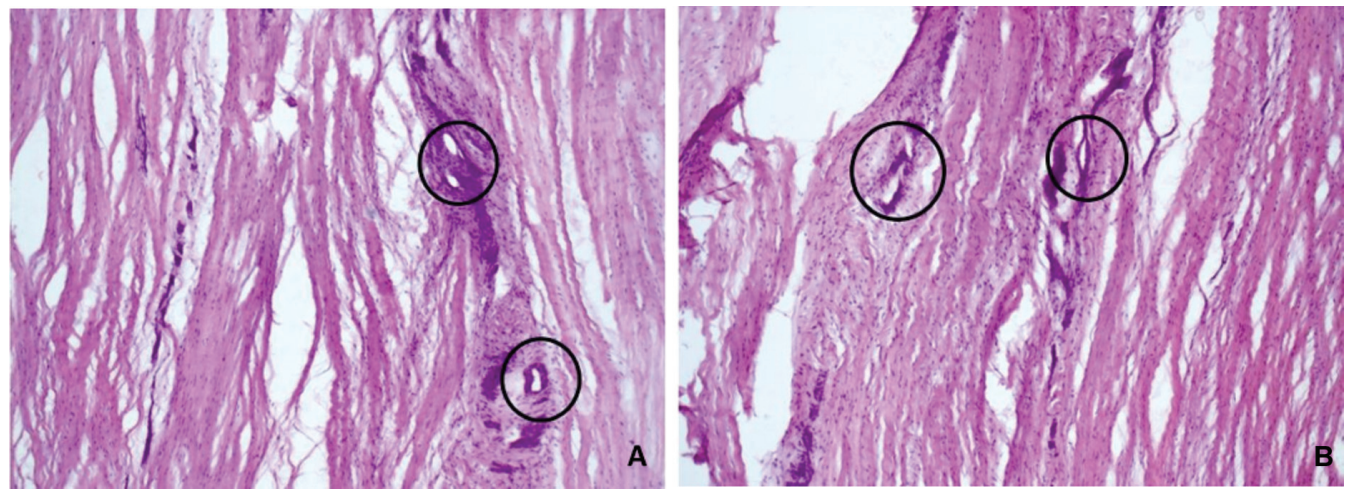

Fig. 1 (A e B) Seção longitudinal e histológica $(10 \mu \mathrm{m})$ manchada com hematoxilina e eosina. Observe os vasos sanguíneos (círculos) no tendão do bíceps.

achados e a fisiopatologia da SLAP e a tendinopatia da cabeça longa da do bíceps.

\section{Métodos}

Foram utilizados 6 complexos bíceps-labrais superiores (SLBCs, na sigla em inglês) de cadáveres humanos congelados (3 homens, 3 mulheres) com idades entre 20 e 70 anos. A aprovação deste projeto foi concedida e acompanhada por meio de nossa institucional pelo comitê de ética ( ${ }^{\circ} 443.172$ ). Utilizamos um marcador pan-neuronal seletivo, o protein gene product 9.5 (PGP 9.5) pan-axonal (Thermo Fisher Scientific Inc., Rockford, IL, EUA), para destacar a inervação sensorial. Os trechos foram lavados com solução tampão de fosfato frio de 0,1 M (PBS, Laborclin, Pinhais, PR, Brasil) contendo 3\% Triton X-100 (TX-100, Inlab, Diadema, SP, Brasil). Os tecidos foram lavados e incubados em anticorpos primários por 2 horas; depois, o anticorpo secundário foi conjugado a uma tag fluorescente (Alexa Fluor 488 goat antirabbit IgG, Thermo Fisher Scientific Inc.). As seções foram lavadas, seladas e os slides foram armazenados a $-70^{\circ} \mathrm{C}$. Todas as 36 seções dos espécimes foram examinadas com um sistema de microscópio de varredura a laser confocal (LSM710, Carl Zeiss Microscopy, Jena, Alemanha). Usamos hematoxilina \& eosina e a mancha tricromática de Masson nos espécimes de SLBC de porção média, e o nervo mediano serviu como controle de qualidade para imunofluorescência. Os resultados são expressos como meios e desvio padrão (DP). Os dados foram analisados usando o software GraphPad
Prism (versão 6.0 para Windows, GraphPad Software, San Diego, Califórnia, EUA).

\section{Resultados}

A análise leve da microscopia das seções mostrou facilmente o tendão do bíceps com vasos sanguíneos escassos, espaçados, finos e ligeiramente ondulados (- Fig. 1, A e B). Na parte de transição entre o bíceps e o labrum, pudemos observar estruturas complexas, com células também alongadas sugerindo células nervosas (-Fig. 2 A, B e C).

O microscópio confocal revelou terminações nervosas livres através da cabeça longa do tendão do bíceps, de 1 a $6 \mu \mathrm{m}$ de comprimento, em paralelo aos feixes de colágeno dispostos, também dissociados da presença de vasos sanguíneos (- Fig.3). Na porção média do SLBC, identificamos fibras nervosas medindo entre 60 e $70 \mu \mathrm{m}$ de diâmetro e ramificando-se em feixes nervosos menores (-Fig. 4).

Ademais, na porção média do SLBC, também se observou fibras nervosas de menor calibre, de 7 a $10 \mu \mathrm{m}$ de diâmetro, próximas ao vaso, exibindo imunorreatividade periférica e intraluminal para PGP 9.5. Nos slides submetidos à técnica de recuperação antigênica, a presença de terminações nervosas complexas com dimensões variáveis, variando de 150 a $350 \mu \mathrm{m}$ de comprimento e 80 a $100 \mu \mathrm{m}$ de largura. Observou-se na face articular das amostras, ao lado da região labral e da transição labrum-bíceps, axônios entre 10 e $20 \mu \mathrm{m}$ de espessura, e diferentes formatos espaciais, com predominância de formas de fuso, cônico e oval (-Fig. 5 A, B e C).
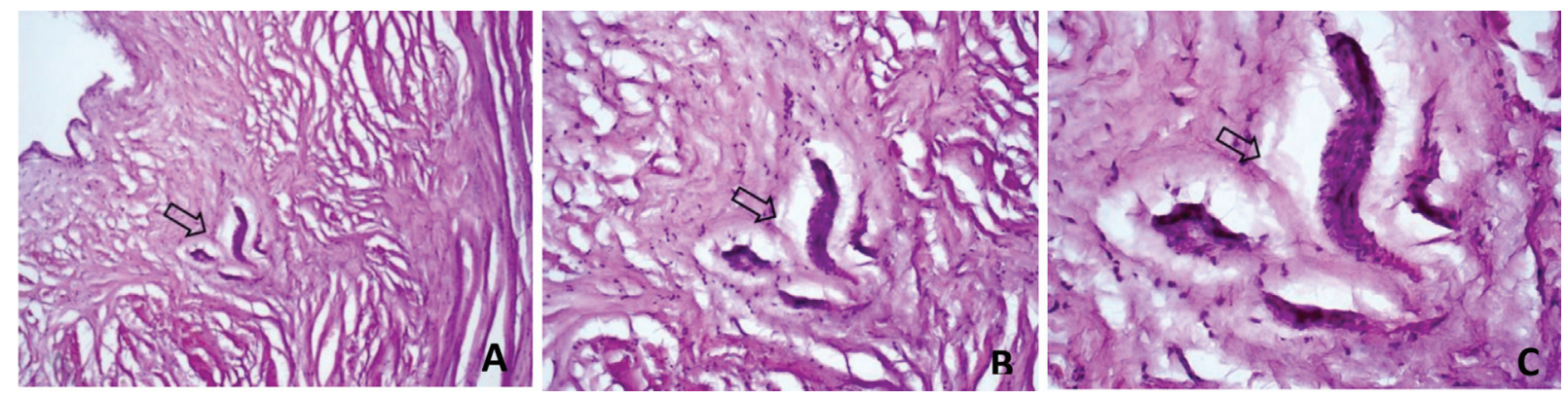

Fig. 2 Terminações nervosas de tecido conjuntivo fusiformes e cônicas na zona de transição entre o labrum e o bíceps. Aumento de 50x (A), 100x (B) e 200x (C). Seção histológica longitudinal $(10 \mu \mathrm{m})$ manchada com hematoxilina e eosina. 


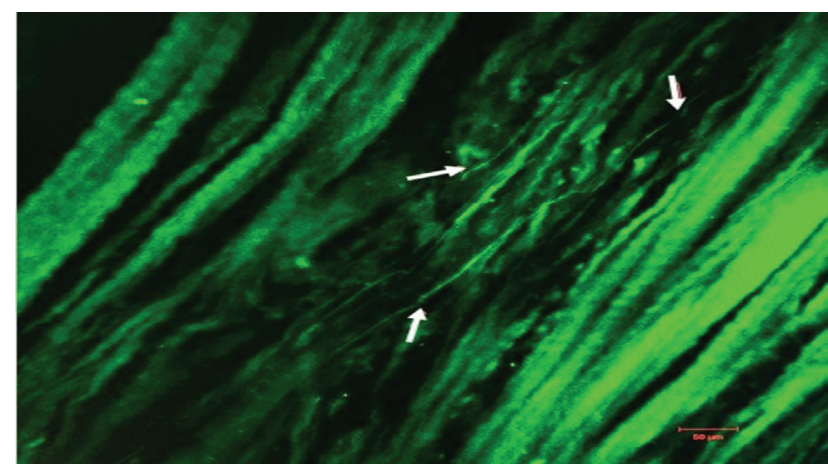

Fig. 3 Seção do tendão do bíceps ( $50 \mu \mathrm{m}$ de espessura). Observe as terminações nervosas livres (setas) no tecido conjuntivo, analisadas pelo microscópio laser confocal (imunofluorescência, escala $50 \mu \mathrm{m}$ ).

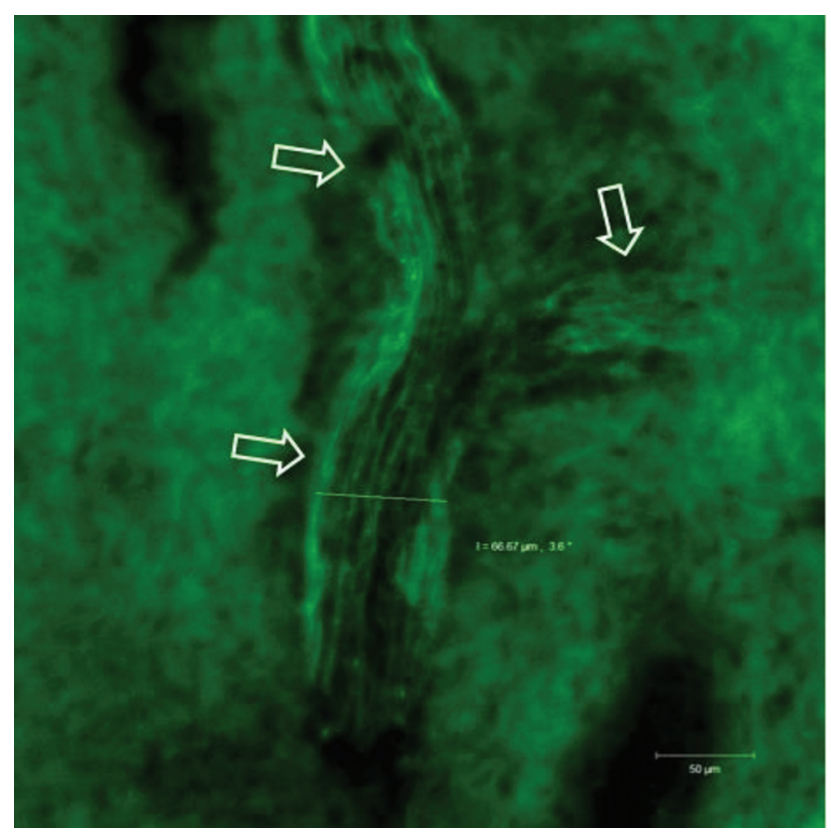

Fig. 4 Zona de transição entre o labrum e o bíceps. Observe a fibra nervosa na camada profunda medindo entre 60 e $70 \mu \mathrm{m}$ de diâmetro, bifurcando em feixes nervosos menores (imunofluorescência, escala de $50 \mu \mathrm{m}$ ).

\section{Discussão}

O conhecimento da neuroanatomia das estruturas estabilizadoras passivas do ombro ajuda a compreender os mecanismos proprioceptivos de proteção e estabilização articular. O tendão da cabeça longa do bíceps tem sido estudado como uma causa de dor na articulação glenoumeral, seja em tendinopatias. ${ }^{15}$ Alpantaki et al. ${ }^{16}$ foram os primeiros a estudar esses elementos neurais no tendão longo do bíceps, que descreveram como contendo uma grande rede de fibras e sensores nervosos simpáticos, não associados aos vasos sanguíneos, e com distribuição neural predominantemente próxima à sua inserção. Nossos achados foram parcialmente compatíveis com os relatados por Alpantaki et al. ${ }^{16}$ Encontramos algumas fibras nervosas finas, seguindo seus próprios caminhos, isoladas dos vasos e dispersas ao longo da estrutura de fibra de colágeno. Observou-se também, proximalmente, fibras maiores na transição labrum-bíceps em torno de estruturas vasculares, como relatado por Boesmueller et al., ${ }^{17}$ que também demonstraram uma densidade de nervos no segmento proximal do tendão do bíceps longo, semelhante à porção anterior do labrum superior. De acordo com esses autores, observou-se a presença de estruturas neurais ocorrendo predominantemente na porção mais proximal do tendão do bíceps. Nas amostras submetidas à técnica de recuperação antigênica, observamos terminações nervosas complexas próximas à porção labral e à transição labrumbicipital, que são predominantemente distribuídas nas camadas mais próximas da articulação glenoide, tornandose possivelmente a primeira região a ser estimulada pelo contato com a cabeça umeral durante o movimento do ombro. Existem informações sobre a localização de estruturas neurais no labrum superior e âncora bicipital. Entre as descrições, Hashimoto et al. ${ }^{18}$ apresentaram a presença isolada de terminações nervosas livres no labrum e transição capsular; segundo Vangsness et al., ${ }^{8}$ temos apenas terminações nervosas livres; Witherspoon et al. ${ }^{9}$ descrevem apenas fascículos nervosos na periferia do labrum anteroinferior e posteroinferior. Em relação ao aspecto fisiopatológico da SLAP no ombro, parece evidente que o tendão bicipital atua como um potencial gerador de dor, tendo maior densidade de estruturas neurais (neurofilamentos) nas partes proximais, como observado em outros estudos. ${ }^{17,19,20}$ Identificamos terminações nervosas nas três regiões (tendão do bíceps, transição labrum e bíceps, labrum superior) do complexo formado pelo labrum superior e pela inserção bicipital, com aspectos bem diferenciados. No segmento proximal do tendão do bíceps, encontramos terminações nervosas livres finas, sem associação com estruturas vasculares, e não foram identificadas terminações nervosas
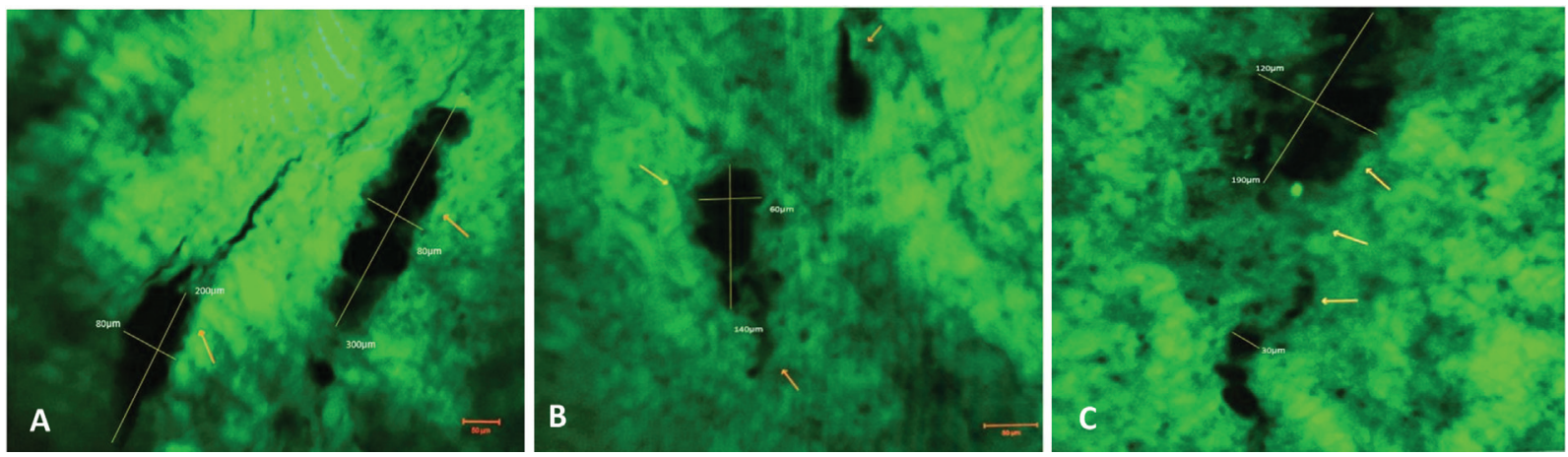

Fig. 5 Terminações nervosas com formas diferentes, variando de 150 a $350 \mu \mathrm{m}$ de comprimento por 80 a $100 \mu \mathrm{m}$ de largura (técnica de recuperação antigênica, escala $50 \mu \mathrm{m}$ ). 
complexas. Na zona de transição entre o labrum e os bíceps, encontramos feixes nervosos espessos, acompanhando os vasos sanguíneos arteriais presentes nesta área. Na região labral e na transição labrum-bicipital, encontramos terminações nervosas complexas estruturas ovais, cônicas e fusiformes. ${ }^{21}$ Há necessidade de novos estudos para confirmar nossos achados, bem como para identificar com precisão os mecanorreceptores, incluindo o teste de outros anticorpos e marcadores celulares, aumentando assim o número de indivíduos na amostra, buscando compreender todos os fatores relacionados à interação da biomecânica e ao sistema proprioceptivo do ombro. 0 presente estudo preliminar mostra a morfologia das terminações nervosas, assim como identifica com precisão os mecanorreceptores por imunofluorescência. No entanto, o número de espécimes e incluindo outros anticorpos e marcadores celulares, seria interessante comparar as condições anatômicas e patológicas.

\section{Conclusão}

Foram identificadas terminações nervosas nas três regiões (tendão do bíceps, transição labrum e bíceps, labrum superior) do complexo formado pelo labrum superior e pela inserção bicipital, com aspectos bem diferenciados. No segmento proximal do tendão do bíceps, encontramos terminações nervosas livres finas, sem associação com estruturas vasculares, e não foram identificadas terminações nervosas complexas. Na zona de transição entre o labrum e o bíceps, encontramos feixes nervosos espessos, acompanhando os vasos sanguíneos arteriais presentes nesta área. Na região labral e na transição labrum-bicipital, encontramos terminações nervosas complexas, sendo possível identificá-las em relação ao formato espacial, que consistiu em estruturas ovais, cônicas e fusiformes.

\section{Contribuições dos autores}

Eduardo G. Fernandes foi o responsável pela concepção, design, conteúdo intelectual e científico do estudo, aquisição e interpretação de dados e redação de manuscritos. M. L. C. Cavalcante foi responsável pela pesquisa, edição de manuscritos, interpretação dos dados e revisão crítica, e submissão do manuscrito. F. V. Jamacaru, E. G. Fernandes, J. V. V. Coelho e J. A. D. Leite estiveram envolvidos nos procedimentos técnicos.

\section{Suporte Financeiro}

Este trabalho teve suporte do Instituto de Pesquisa e Ensino Home, Brasília, DF, Brasil.

Conflito de Interesses

Os autores não têm conflito de interesses para declarar.

\section{Referências}

1 Werner BC, Brockmeier SF, Miller MD. Etiology, Diagnosis, and Management of Failed SLAP Repair. J Am Acad Orthop Surg 2014; 22(09):554-565
2 Andrews JR, Carson WG Jr, McLeod WD. Glenoid labrum tears related to the long head of the biceps. Am J Sports Med 1985;13 (05):337-341

3 Snyder SJ, Karzel RP, Del Pizzo W, Ferkel RD, Friedman MJ. SLAP lesions of the shoulder. Arthroscopy 1990;6(04):274-279

4 Maffet MW, Gartsman GM, Moseley B. Superior labrum-biceps tendon complex lesions of the shoulder. Am J Sports Med 1995;23 (01):93-98

5 Boesmueller S, Mayerhofer S, Huf W, Fialka C. Short-term clinical results after arthroscopic type II SLAP repair. Wien Klin Wochenschr 2012;124(11-12):370-376

6 Katz LM, Hsu S, Miller SL, et al. Poor outcomes after SLAP repair: descriptive analysis and prognosis. Arthroscopy 2009;25(08): 849-855

7 Boileau P, Baqué F, Valerio L, Ahrens P, Chuinard C, Trojani C. Isolated arthroscopic biceps tenotomy or tenodesis improves symptoms in patients with massive irreparable rotator cuff tears. J Bone Joint Surg Am 2007;89(04):747-757

8 Vangsness CT Jr, Ennis M, Taylor JG, Atkinson R. Neural anatomy of the glenohumeral ligaments, labrum, and subacromial bursa. Arthroscopy 1995;11(02):180-184

9 Witherspoon JW, Smirnova IV, McIff TE. Neuroanatomical distribution of mechanoreceptors in the human cadaveric shoulder capsule and labrum. J Anat 2014;225(03):337-345

10 Warner JJ, Lephart S, Fu FH. Role of proprioception in pathoetiology of shoulder instability. Clin Orthop Relat Res 1996;(330):35-39

11 Onyekwelu I, Khatib O, Zuckerman JD, Rokito AS, Kwon YW. The rising incidence of arthroscopic superior labrum anterior and posterior (SLAP) repairs. J Shoulder Elbow Surg 2012;21(06): 728-731

12 Brodin L, Ericsson M, Mossberg K, Hökfelt T, Ohta Y, Grillner S. Three-dimensional reconstruction of transmitter-identified central neurons by "en bloc" immunofluorescence histochemistry and confocal scanning microscopy. Exp Brain Res 1988;73(02):441-446

13 Chen YG, McClinton MA, DaSilva MF, Shaw Wilgis EF. Innervation of the metacarpophalangeal and interphalangeal joints: a microanatomic and histologic study of the nerve endings. J Hand Surg Am 2000;25(01):128-133

14 Vilensky JA, O'Connor BL, Fortin JD, et al. Histologic analysis of neural elements in the human sacroiliac joint. Spine 2002;27(11): 1202-1207

15 Longo UG, Franceschi F, Ruzzini L, et al. Characteristics at haematoxylin and eosin staining of ruptures of the long head of the biceps tendon. Br J Sports Med 2009;43(08):603-607

16 Alpantaki K, McLaughlin D, Karagogeos D, Hadjipavlou A, Kontakis G. Sympathetic and sensory neural elements in the tendon of the long head of the biceps. J Bone Joint Surg Am 2005;87(07): 1580-1583

17 Boesmueller S, Nógrádi A, Heimel P, et al. Neurofilament distribution in the superior labrum and the long head of the biceps tendon. J Orthop Surg Res 2017;12(01):181

18 Hashimoto T, Hamada T, Sasaguri Y, Suzuki K. Immunohistochemical approach for the investigation of nerve distribution in the shoulder joint capsule. Clin Orthop Relat Res 1994;(305): 273-282

19 Joseph M, Maresh CM, McCarthy MB, et al. Histological and molecular analysis of the biceps tendon long head post-tenotomy. J Orthop Res 2009;27(10):1379-1385

20 Tosounidis T, Hadjileontis C, Georgiadis M, Kafanas A, Kontakis G. The tendon of the long head of the biceps in complex proximal humerus fractures: a histological perspective. Injury 2010;41 (03):273-278

21 Tomita K, Berger EJ, Berger RA, Kraisarin J, An KN. Distribution of nerve endings in the human dorsal radiocarpal ligament. J Hand Surg Am 2007;32(04):466-473 